\title{
Um estudo comparativo entre dados sociodemográficos e neuroticismo ${ }^{1}$
}

\section{A comparative study among sociodemographic data and neuroticism}

\section{Mansueto Dal Maso*}

Faculdade de Ciências Biomédicas de Cacoal - FACIMED, Cacoal, Rondônia, Brasil

\section{Fabio Biasotto Feitosa**}

Universidade Federal de Rondônia - UNIR, Porto Velho, Rondônia, Brasil

\begin{abstract}
RESUMO
O neuroticismo tem sido referido como um importante indicador de saúde mental. O estudo do grau de neuroticismo em uma dada população pode ser estratégico para o planejamento de políticas e programas de saúde coletiva. O objetivo do presente estudo foi avaliar o neuroticismo em estudantes universitários do Estado de Rondônia. Participaram como sujeitos deste estudo de levantamento 1.031 universitários de ambos os sexos, entre 18 e 75 anos, que autoavaliaram seu grau de neuroticismo. Os dados foram coletados mediante uso de um formulário com itens sociodemográficos e da Escala Fatorial de Neuroticismo - EFN. Os resultados mostraram que o neuroticismo variou significativamente conforme o sexo e o estado civil. Discutem-se as implicações teóricas e práticas do neuroticismo para a atuação psicológica em saúde coletiva.
\end{abstract}

Palavras-chave: Neuroticismo, Depressão, Coping.

\section{ABSTRACT}

Neuroticism has been refered as an important indicator of mental health. The study of the neuroticism level in a specific population can be strategic to plan politics and collective health programs. The aim of this study was to assess the neuroticism in college students from Rondonia. The subjects that participated in this survey study were 1.031 students of both genders, between 18-75 years-old, who self-evaluated the level of their neuroticism. Data were collected by using a form with sociodemographic items and the EFN to assess neuroticism. Results showed that neuroticism levels varied significantly according to sex and marital status. The theorical and practical implications of the neuroticism for psychological work in collective health were discussed.

Keywords: Neuroticism, Depression, Coping.

\section{I ntrodução}


O neuroticismo é um traço da personalidade conceituado como uma tendência relativamente estável a responder com emoções negativas às frustrações e ameaças (LAHEY, 2009). Um alto nível de neuroticismo caracteriza-se pela propensão a vivenciar mais intensamente sofrimento emocional (HUTZ; NUNES, 2001), o que o torna um indicador significativo de saúde mental. $O$ estudo de Weinstock e Whisman (2006) mostrou que pessoas com ansiedade e depressão tendem a reportar escores elevados em testes de neuroticismo. Conforme Watson e Casillas (2003), o neuroticismo tende a estar mais associado a psicopatologias do que qualquer outro traço de personalidade.

Segundo Lesch (2003), há um corpo considerável de evidências indicando que o neuroticismo, abrangendo um amplo espectro da ansiedade, é influenciado por complexos componentes genéticos. 0 estudo de Viken, Rose, Kaprio e Koskenvuo (1994), com quase 15.000 gêmeos finlandeses, sustentou que a genética é nas mulheres a primeira fonte da estabilidade do neuroticismo ao longo da vida. No estudo de Schneewind e Kupsch (2007) com 632 casais alemães, austríacos e suíços, as mulheres relataram apresentar grau mais elevado de neuroticismo em comparação aos homens. Além disso, nesse estudo, homens e mulheres, com graus mais elevados de neuroticismo e percepção de menos recursos pessoais e sociais para o enfrentamento das adversidades do cotidiano, apresentaram maior percepção de estresse.

O neuroticismo é também considerado uma estratégia de adaptação, sendo ativado ou desativado conforme as circunstâncias de vida que representam adversidade ou segurança (WATSON; CASILLAS, 2003). Sendo assim, neuroticismo baixo pode significar uma tendência a não evitar riscos desnecessários, podendo ser tão prejudicial quanto o neuroticismo intenso, embora o significado do neuroticismo elevado seja mais conhecido (HUTZ; NUNES, 2001).

\section{Neuroticismo e bem-estar}

O estudo brasileiro de Nunes, Hutz e Giacomoni (2009), com 357 estudantes universitários, mostrou correlações significativas e invertidas entre neuroticismo e satisfação com a vida $(r=-0,49$; $p<0,01)$, neuroticismo e afeto positivo $(r=-0,27 ; p<0,01)$, bem como correlação positiva significativa entre neuroticismo e afeto negativo $(r=0,44 ; p<0,01)$. O neuroticismo foi acessado por meio de quatro subescalas que avaliaram componentes subjetivos relacionados à vulnerabilidade, ao desajustamento psicossocial, à ansiedade e à depressão nesses estudantes. No referido estudo, a depressão foi a subescala de maior correlação inversa com satisfação na vida ( $r=-$ $0,56 ; p<0,01)$. 
No estudo de Gunthert, Cohen e Armeli (1999), universitários ( $\mathrm{N}=197)$ completaram questionários no final de cada dia durante 14 dias consecutivos, registrando o evento mais estressante do dia e suas reações a eles. Os estudantes com elevado neuroticismo, comparados aos estudantes com neuroticismo baixo, relataram maiores estressores interpessoais, acompanhados de mais percepções negativas e formas menos adaptativas de enfrentamento (exemplo: hostilidade).

Em resumo, os eventos de vida podem afetar o grau de neuroticismo (SCOLLON; DIENER, 2006), assim como o neuroticismo pode influenciar a forma de vivenciar tais eventos (GUNTHERT et al., 1999) ou mesmo predispor o indivíduo à ocorrência de eventos negativos objetivos (MAGNUS; DIENER; FUJITA; PAVOT, 1993). Evidencia-se que o neuroticismo tem significativas implicações para a avaliação da saúde mental e do bem-estar das pessoas, bem como para a saúde coletiva (LAHEY, 2009), chamando a atenção para a pertinência da avaliação do neuroticismo em população universitária.

De acordo com Vendramini et al. (2004), tem sido cada vez mais evidente a preocupação de pesquisadores em relação à integração do estudante à vida universitária. Segundo as autoras, o processo de socialização dos estudantes universitários dependerá em parte do seu grau de vulnerabilidade, da capacidade adaptativa e de enfrentamento. Podem ser citados, como desafios para muitos estudantes, a saída de casa, a separação da família e dos amigos, a saída da sua cidade e as exigências do novo contexto educativo (ALMEIDA; SOARES; FERREIRA, 2002), exigindo a reformulação dos relacionamentos interpessoais e familiares, o estabelecimento de novas amizades, a redefinição do papel de estudante, o gerenciamento do tempo e atividades no quadro de uma maior autonomia (ALMEIDA; GUISANDE; SOARES; SAAVEDRA, 2006). Essas alterações podem desencadear maiores ou menores níveis de estresse que, em certos casos, conduzem ao desajustamento, fracasso e abandono acadêmicos (ALMEIDA et al., 2006).

Nessa direção, salienta-se que a assistência adequada às demandas dos universitários requer o levantamento prévio de suas condições de saúde e necessidades psicológicas, a fim de possibilitar a organização e o planejamento de programas assistenciais coletivos específicos a essa população (SERPA; SANTOS, 2001). No entanto, expõe Gottlieb (2000), embora a necessidade desse tipo de levantamento diagnóstico seja óbvia, na promoção da saúde raramente esse cuidado é tomado, uma vez que, geralmente, são os usuários que se adaptam aos programas de saúde ao invés destes serem adaptados às necessidades de apoio percebidas pelos usuários. Seria pertinente, nesse contexto, verificar as possíveis variações do grau de neuroticismo na relação com variáveis sociodemográficas, como o estado civil e o sexo, a idade e o nível socioeconômico, para o 
levantamento de demandas psicológicas prioritárias em populações específicas. O psicólogo, por meio de pesquisas avaliativas e de levantamento, poderá, dessa forma, referenciar futuros programas de saúde junto a estudantes universitários.

Diante disso, considerando (1) as correlações entre neuroticismo, saúde mental e bem-estar (LAHEY, 2009; NUNES et al., 2009), (2) o envolvimento do neuroticismo nos processos de adaptação ou enfrentamento das adversidades (WATSON; CASILLAS, 2003), e (3) a necessidade de estudos de levantamento para a avaliação de demandas psicológicas de uma população (GOTTLIEB, 2000), com especial atenção às demandas de estudantes universitários (SERPA; SANTOS, 2001), o objetivo do presente estudo foi avaliar o neuroticismo em estudantes universitários do Estado de Rondônia.

\section{Método}

O presente estudo consistiu de uma pesquisa de levantamento, com obtenção de uma grande amostra a partir de uma população de interesse para posterior análise estatística descritiva e inferencial (ANASTASI, 1961/1977; BUNCHAFT; CAVAS, 2002; STURGIS, 2010).

\subsection{Participantes}

Participaram como sujeitos desta pesquisa 1.031 estudantes universitários de ambos os sexos (Masculino $=32 \%$, Feminino $=68 \%$ ), entre 18 e 75 anos (Mediana=22; Média=24,5; Desvio Padrão=6,9), do Estado de Rondônia. Como critérios de inclusão na composição da amostra, os estudantes universitários deveriam ter no mínimo dezoito anos de idade e aceitar participar voluntariamente. Foram excluídos da amostra os casos com erros no preenchimento da EFN, tais como duplas respostas ou itens em branco, que por algum motivo não foram corrigidos no momento da coleta. Os casos com omissões no preenchimento de itens dos dados sociodemográficos foram reduzidos e, por isso, foram incluídos na amostra e indicados na seção de resultados.

\subsection{I nstrumentos}

Escala Fatorial de Neuroticismo (EFN). A EFN (HUTZ; NUNES, 2001) é um instrumento clínico para indicação de padrões emocionais associados ao desconforto psicológico, frequentemente presentes em transtornos de personalidade, em indivíduos jovens e adultos. Apresenta 82 itens e respostas em Likert de sete pontos, na qual 7 corresponde a frase que descreve muito bem opiniões, sentimentos ou atitudes do sujeito, e 1 quando absolutamente não descreve bem. 
A EFN oferece normas de interpretação obtidas em uma amostra de 1.176 universitários de sete estados brasileiros (RS, SC, BA, PE, RJ, PB, MG). De maneira abrangente, permite uma avaliação de importantes aspectos da personalidade humana, a partir da Escala Geral e dos Fatores (F) ou Subescalas: F1-Vulnerabilidade (medo de críticas, insegurança, baixa autoestima, dificuldade de tomar decisões, medo de abandono das pessoas mais próximas), F2Desajustamento psicossocial (comportamentos sexuais de risco ou atípicos, adição ou consumo exagerado de álcool, hostilidade com pessoas ou animais, necessidade recorrente em chamar a atenção), F3-Ansiedade (sintomas somáticos relacionados com ansiedade, irritabilidade, transtornos do sono, impulsividade, sintomas de pânico, mudanças de humor), e F4-Depressão (itens relacionados com escalas de depressão, suicídio e desesperança). Embora não ofereça um sistema para discriminação de casos dentro de concepções de normalidade e patologia, sugere-se que escores muito altos ou muito baixos devam indicar alguns tipos de transtornos de personalidade, exigindo uma investigação mais profunda. A partir dos escores brutos é possível apurar o escore padrão da Escala Geral, que foi dimensionada para ter uma média de 100 pontos e um desvio padrão de 20, de maneira que escores entre 80 e 120 são esperados para a maior parte da população. O instrumento, validado em amostra brasileira, apresenta boa consistência interna (Alpha de Cronbach $=0,94$ ).

Formulário com itens sociodemográficos. Elaborado a partir do Critério de Classificação Econômica Brasil-CCEB (IBOPE/ABEP, 2004), com adaptações para autoaplicação, forneceu dados pessoais e caracterizou a amostra em termos socioeconômicos. O CCEB divide a população brasileira em cinco classes de poder aquisitivo, em ordem decrescente, compreendendo: A, B, C, D e E. A classificação do poder aquisitivo, conforme os critérios padrões, é feita mediante a atribuição de escores para a presença de bens de consumo no lar, empregados domésticos, nível de escolarização do chefe da família etc. O presente estudo observou os critérios do CCEB para a classificação do nível socioeconômico dos participantes, mas transformou o formulário original, projetado para ser aplicado por um entrevistador, em um instrumento de autoaplicação. Além disso, o formulário incluiu diferentes itens sociodemográficos, tais como tempo de moradia em Rondônia, cidade, curso e instituição onde estuda, estado civil, sexo e faixa etária do respondente.

\subsection{Procedimentos}

A coleta de dados foi realizada no biênio 2008-2009 em seis Instituições de Ensino Superior (IES) do Estado de Rondônia que aceitaram participar oferecendo autorização por escrito para a 
pesquisa ser realizada junto aos estudantes em sala de aula, sendo duas instituições da capital e as outras quatro do interior. Em cada instituição a coleta de dados foi realizada de maneira coletiva por um professor pesquisador, assessorado por estudantes de psicologia, respeitando-se os horários mais convenientes para a instituição e para os professores das turmas envolvidas. Os estudantes de psicologia que assessoraram o pesquisador receberam dele treinamento específico para a aplicação dos instrumentos. Após explicação da pesquisa, seus objetivos e esclarecimentos de dúvidas, os estudantes em sala de aula que aceitaram participar como sujeitos da pesquisa assinaram o Termo de Consentimento Livre e Esclarecido (TCLE) e responderam aos instrumentos de coleta de dados. Ao término dos preenchimentos, o pesquisador e seus assessores conferiram omissões e duplos preenchimentos nas respostas, solicitando do participante complementações e correções quando necessário. Os dados foram tabulados e submetidos a análises estatísticas, observando o sigilo dos participantes. Conforme as normas do Conselho Nacional de Saúde (Resolução 196/96), o projeto do qual derivou a presente pesquisa foi submetido à apreciação do CEP - Comitê de Ética em Pesquisa da FACIMED e aprovado.

\section{Resultados}

Considerando as indicações de autores (FIFE-SCHAW, 2010; PASQUALI, 2010) para escalas ordinais, optou-se no presente estudo pelo uso de testes não paramétricos para as análises descritivas e inferenciais. Os escores utilizados nas análises estatísticas foram os escores brutos. Assim, verificou-se que os estudantes universitários pesquisados no Estado de Rondônia estavam em cursos de graduação em IES privadas $(81 \%)$ e públicas $(19 \%)^{2}$, e declararam morar em Rondônia entre menos de um ano a até 53 anos (Mediana=20; Média=19,8; Desvio Padrão=7,4).

Observou-se que a maioria dos estudantes universitários pesquisados era do sexo feminino (68\%) e, subdividindo a amostra total em faixas etárias, foi encontrado que $53,6 \%$ deles tinham entre 18 e 22 anos, $21,1 \%$ entre 23 e 27 anos, $12,1 \%$ entre 28 e 32 anos e $13 \%$ tinham 33 anos ou mais (apenas dois participantes da amostra não quiseram informar a idade). Quanto ao estado civil, 69,7\% dos estudantes eram solteiros, sendo que $25,3 \%$ eram casados ou em união estável e $1,7 \%$ eram viúvos ou divorciados ( $3,3 \%$ não quiseram informar 0 seu estado civil). A avaliação do nível socioeconômico da amostra revelou que $6,7 \%$ foi classificada no nível $A, 47,8 \%$ pertencia à classe $B, 40,9 \%$ foi classificada no nível $C$ e $4,5 \%$ pertencia à classe $D$ 
(apenas um participante da amostra não quis informar seu nível socioeconômico).

Os universitários pesquisados estavam em sua maioria estudando no interior do estado $(62,4 \%)$, compreendendo os municípios de Rolim de Moura (18,3\%), Cacoal $(25,3 \%)$ e Ariquemes (18,8\%), enquanto $37,6 \%$ deles estudavam na capital, Porto Velho. Quanto à area de estudos, $46 \%$ dos participantes da pesquisa estavam em cursos das Ciências Biológicas, 37,6\% em cursos das Ciências Sociais e 16,4\% em cursos das Ciências Exatas.

Os escores na EFN foram comparados considerando o estado civil e o sexo dos estudantes universitários. A Tabela 1 aponta diferenças significativas entre universitários casados e solteiros $(p<0,001)$, bem como entre homens e mulheres $(p<0,01)$, de maneira que os solteiros, em relação aos casados, e as mulheres, em relação aos homens, relataram escores gerais mais altos de neuroticismo. As mesmas análises comparativas não foram procedidas com as outras categorias de estado civil (divorciado, viúvo) devido ao número muito reduzido de casos na amostra.

Tabela 1. Comparativo da Escala Geral de neuroticismo conforme o estado avil e o sexo.

\begin{tabular}{ccccccc}
\hline Variáveis & N & Média & D.P. & Rank & Mann-Whitney U & Sig. \\
\hline Estado Civil & & & & & & \\
$\quad \begin{array}{c}\text { Solteiro } \\
\text { Casado }\end{array}$ & 719 & 191,9 & 59,0 & 514,3 & 75990,0 & 0,000 \\
$\quad \begin{array}{l}\text { Sexo } \\
\text { Masculino }\end{array}$ & 331 & 174,9 & 55,2 & 422,8 & & \\
Feminino & 700 & 190,6 & 55,9 & 480,0 & 103924,5 & 0,008 \\
\hline
\end{tabular}

Uma vez que houve diferenças significativas para o sexo e o estado civil, foram detalhadas as análises, primeiramente comparando-se os homens $e$ as mulheres pelas subescalas ou fatores $(F)$ de neuroticismo conforme o seu estado civil. Como apresentado na Tabela 2, os resultados revelaram que as mulheres solteiras relataram, comparando-se aos homens solteiros, maiores escores em Vulnerabilidade $(p<0,01)$ e Ansiedade $(p<0,001)$. Contudo, os homens solteiros relataram, comparando-se às mulheres solteiras, maiores escores em Desajustamento psicossocial $(p<0,001)$. $\mathrm{Na}$ subescala Depressão não houve diferenças significativas entre mulheres e homens solteiros $(p=0,517)$. Ainda de acordo com a Tabela 2, as mulheres casadas relataram, comparando-se aos homens casados, maiores escores em Vulnerabilidade $(p<0,01)$, 
Ansiedade $(p<0,01)$ e Depressão $(p<0,001)$. Não houve diferenças significativas entre mulheres e homens casados em Desajustamento psicossocial $(p=0,459)$.

Tabela 2. Comparativo do neuroticismo entre homens e mulheres conforme o estado civil.

\begin{tabular}{|c|c|c|c|c|c|c|}
\hline Variáveis & $\mathbf{N}$ & Média & D.P. & Rank & Mann-Whitney U & Sig. \\
\hline \multicolumn{7}{|c|}{$\begin{array}{l}\text { Grupo de Solteiros } \\
\text { F1-Vu/nerabilidade }\end{array}$} \\
\hline Homens & 239 & 60,2 & 21,3 & 330,3 & 50268,0 & 0,007 \\
\hline Mulheres & 480 & 65,5 & 23,7 & 374,8 & & \\
\hline \multicolumn{7}{|c|}{ F2-Desajustamento } \\
\hline Homens & 239 & 23,7 & 10,3 & 403,1 & 47068,5 & 0,000 \\
\hline Mulheres & 480 & 21,0 & 9,0 & 338,6 & & \\
\hline \multicolumn{7}{|l|}{ F3-Ansiedade } \\
\hline Homens & 239 & 60,9 & 23,5 & 316,0 & 46844,5 & 0,000 \\
\hline Mulheres & 480 & 68,3 & 25,0 & 381,9 & & \\
\hline \multicolumn{7}{|l|}{ F4-Depressão } \\
\hline Homens & 239 & 40,7 & 14,4 & 367,1 & 55662,5 & 0,517 \\
\hline Mulheres & 480 & 40,3 & 14,7 & 356,5 & & \\
\hline \multicolumn{7}{|c|}{$\begin{array}{l}\text { Grupo de Casados } \\
\text { F1-Vulnerabilidade }\end{array}$} \\
\hline Homens & 68 & 51,2 & 17,2 & 107,9 & 4991,5 & 0,004 \\
\hline Mulheres & 192 & 61,3 & 24,0 & 138,5 & & \\
\hline \multicolumn{7}{|c|}{ F2-Desajustamento } \\
\hline Homens & 68 & 19,1 & 6,0 & 136,2 & 6139,0 & 0,459 \\
\hline Mulheres & 192 & 18,7 & 6,5 & 128,5 & & \\
\hline \multicolumn{7}{|l|}{ F3-Ansiedade } \\
\hline Homens & 68 & 52,2 & 16,0 & 108,5 & 5031,5 & 0,005 \\
\hline & 192 & 61,5 & 23,0 & 138,3 & & \\
\hline \multicolumn{7}{|l|}{ F4-Depressão } \\
\hline Homens & 68 & 33,9 & 12,6 & 102,4 & 4614,5 & 0,000 \\
\hline Mulheres & 192 & 39,9 & 14,5 & 140,5 & & \\
\hline
\end{tabular}

Depois, foram analisadas pelas subescalas ou fatores $(F)$ de neuroticismo as diferenças entre solteiros e casados em cada sexo, conforme apresenta a Tabela 3. Os homens solteiros, em relação aos homens casados, relataram escores mais elevados de neuroticismo em todas as subescalas Vulnerabilidade $(p<0,01)$, Desajustamento psicossocial $(p<0,001)$, Ansiedade $(p<0,05)$ e Depressão $(p<0,001)$. De maneira semelhante, as mulheres solteiras, em relação às mulheres casadas, relataram escores mais elevados de neuroticismo nas subescalas Vulnerabilidade $(p<0,05)$, Desajustamento psicossocial $(p<0,001)$ e Ansiedade $(p<0,01)$, contudo, não apresentaram diferenças significativas em Depressão $(p=0,717)$. 
Mansueto Dal Maso, Fabio Biasotto Feitosa Um estudo comparativo entre dados sociodemográficos e neuroticismo

Tabela 3. Comparativo do neuroticismo entre solteiros e casados conforme o sexo.

\begin{tabular}{|c|c|c|c|c|c|c|}
\hline Variáveis & $\mathbf{N}$ & Média & D.P. & Rank & Mann-Whitney U & Sig. \\
\hline \multicolumn{7}{|c|}{$\begin{array}{l}\text { Sexo Masculino } \\
\text { F1-Vulnerabilidade }\end{array}$} \\
\hline Solteiros & 239 & 60,2 & 21,3 & 162,5 & 6084,5 & 0,002 \\
\hline Casados & 68 & 51,2 & 17,2 & 124,0 & & \\
\hline \multicolumn{7}{|c|}{ F2-Desajustamento } \\
\hline Solteiros & 239 & 23,7 & 10,3 & 164,5 & 5617,5 & 0,000 \\
\hline $\begin{array}{c}\text { Casados } \\
\text { F3-Ansiedade }\end{array}$ & 68 & 19,1 & 6,0 & 117,1 & F3-Ansiedade & \\
\hline Solteiros & 239 & 60,9 & 23,5 & 160,9 & 6478,0 & 0,011 \\
\hline Casados & 68 & 52,2 & 16,0 & 129,8 & & \\
\hline \multicolumn{7}{|l|}{ F4-Depressão } \\
\hline Solteiros & 239 & 40,7 & 14,4 & 166,0 & 5263,5 & 0,000 \\
\hline Casados & 68 & 33,9 & 12,6 & 111,9 & & \\
\hline \multicolumn{7}{|c|}{$\begin{array}{l}\text { Sexo Feminino } \\
\text { F1-Vulnerabilidade }\end{array}$} \\
\hline Solteiras & 480 & 65,5 & 23,7 & 347,3 & 40876,0 & 0,022 \\
\hline Casadas & 192 & 61,3 & 24,0 & 309,4 & & \\
\hline \multicolumn{7}{|c|}{ F2-Desajustamento } \\
\hline Solteiras & 480 & 21,0 & 9,0 & 355,5 & 36972,5 & 0,000 \\
\hline \multirow{2}{*}{\multicolumn{7}{|c|}{ F3-Ansiedade }} \\
\hline & & & & & & \\
\hline Solteiras & 480 & 68,3 & 25,0 & 352,2 & 38565,5 & 0,001 \\
\hline Casadas & 192 & 61,5 & 23,0 & 297,4 & & \\
\hline \multicolumn{7}{|l|}{ F4-Depressão } \\
\hline Solteiras & 480 & 40,3 & 14,7 & 338,2 & 45255,0 & 0,717 \\
\hline Casadas & 192 & 39,9 & 14,5 & 332,2 & & \\
\hline
\end{tabular}

A Tabela 4 apresenta o escore padrão da Escala Geral de neuroticismo, apontando que o desempenho da amostra tanto masculina $(89,9)$ quanto feminina $(90,3)$ na EFN foi médio-inferior, porém acima dos 80 pontos limítrofes, possibilitando concluir que houve uma tendência geral para 0 relato de neuroticismo de moderado a baixo. 
Tabela 4. Escore padrão geral da amostra masculina e feminina na EFN.

\begin{tabular}{ccccc}
\hline Variáveis & N & Mediana & D.P. & Escore Padrão \\
\hline Sexo Masculino & & & & \\
F1-Vulnerabilidade & 331 & 56,0 & 20,9 & 22,0 \\
F2-Desajustamento & 331 & 20,0 & 10,3 & 23,3 \\
F3-Ansiedade & 331 & 54,0 & 22,8 & 21,9 \\
F4-Depressão & 331 & 36,0 & 14,2 & 22,7 \\
EFN-Escala Geral & 331 & 170,0 & 55,9 & 89,9 \\
Sexo Feminino & & & & \\
F1-Vulnerabilidade & 700 & 60,0 & 23,7 & 22,5 \\
F2-Desajustamento & 700 & 17,0 & 8,3 & 21,8 \\
F3-Ansiedade & 700 & 62,0 & 24,6 & 22,0 \\
F4-Depressão & 700 & 37,0 & 14,6 & 24,0 \\
EFN-Escala Geral & 700 & 177,0 & 59,4 & 90,3 \\
\hline
\end{tabular}

O teste de correlação de Spearman foi inicialmente aplicado para verificar a possibilidade do grau de neuroticismo variar em relação à idade e ao nível socioeconômico. Contudo, a correlação inversa do neuroticismo com a idade dos universitários $\left(r_{s}=-0,164 ; p<0,001\right)$ e a correlação positiva do neuroticismo com o nível socioeconômico $\left(r_{s}=0,098 ; p<0,01\right)$ encontradas, embora significativas, foram muito baixas, inviabilizando análises estatísticas mais detalhadas.

\section{Discussão}

O presente estudo avaliou o grau de neuroticismo na população universitária de Rondônia e os resultados ofereceram informações pertinentes à caracterização e compreensão de algumas demandas psicológicas nessa população. No presente estudo, houve uma tendência geral de homens relatarem graus mais baixos de neuroticismo, quando comparados às mulheres, em consonância com o estudo de Schneewind e Kupsch (2007). Uma vez que as mulheres relataram um nível de neuroticismo mais elevado em relação aos homens, pode-se levantar a hipótese de que essas mulheres constituem um grupo com maior risco ao adoecimento por estresse ou sofrimento emocional.

No entanto, o presente estudo mostrou que os homens relataram escores mais elevados em desajustamento psicossocial, à semelhança do estudo de Hutz e Nunes (2001), mas, há de se notar que o resultado ocorreu somente no grupo de estudantes solteiros. As condições e responsabilidades próprias do casamento poderiam 
explicar a tendência mais reduzida ao desajustamento psicossocial nos homens casados. De acordo com Reis e Collins (2000), o suporte social, que é protetor da saúde, dependeria de relacionamentos de maior proximidade (close relationships). Dessa forma, o suporte social, disponibilizado, nesses casos, pela companheira, protegeria a saúde dos homens ao contribuir com a redução do desajustamento psicossocial. Corrobora com essa visão o fato dos homens solteiros, em relação aos homens casados, relatarem escores mais elevados de neuroticismo nas quatro subescalas, vulnerabilidade, desajustamento, ansiedade e depressão. O mesmo se deu no caso das mulheres solteiras em relação às casadas, com exceção da subescala depressão.

Nota-se, pelos resultados do presente estudo, que a depressão esteve independente do estado civil para o sexo feminino, e dependente do estado civil para o sexo masculino. A depressão na mulher tem sido registrada na literatura como duas vezes mais frequente do que a depressão em homens, independentemente do país e da cultura, possivelmente relacionada a componentes hormonais, aos efeitos de gerar filhos, estressores diferentes para os sexos e modelos comportamentais de desamparo aprendido (SADOCK; SADOCK, 2007). No entanto, a depressão masculina tem sido quase negligenciada pela literatura, de maneira que futuros estudos poderiam investigar as circunstâncias ambientais (familiares, sociais) e psicológicas (afetividade, crenças, atitudes, expectativas) envolvidas na depressão dos homens, especialmente entre os solteiros. Sabe-se, contudo, que a depressão ocorre com maior frequência em pessoas sem relacionamentos interpessoais íntimos (SADOCK; SADOCK, 2007), podendo explicar os casos de universitários masculinos solteiros do presente estudo que não diferiram das mulheres solteiras no item depressão.

Sendo assim, a manifestação do neuroticismo, em forma de depressão, pareceu ser mais um traço na personalidade feminina, envolvendo predominantemente condições psicológicas e biológicas inerentes ao sexo, enquanto que na vivência masculina seria mais um estado, influenciado predominantemente por condições transitórias. 0 amplo estudo de Viken et al. (1994) sustenta essa visão. As respostas cognitivas, afetivas e comportamentais que compõem o construto neuroticismo parecem, diante isso, variar em graus de frequência e intensidade em um continuum que abrangeria desde um ponto de maior rigidez, com características inerentes à personalidade e de origem fortemente genética (LESCH, 2003; VIKEN et al., 1994), ao ponto de maior flexibilidade, isto é, representativo de características flutuantes e dependentes de contingências ambientais (GUNTHERT et al., 1999; SCOLLON; DIENER, 2006). De qualquer forma, o neuroticismo pode ser subsumido em uma maneira peculiar da pessoa vivenciar, com sofrimento, e em dado momento, mas não 
necessariamente limitado ao mesmo, os desafios e adversidades do cotidiano, o que remete a implicações para a intervenção em saúde coletiva.

O neuroticismo tem servido como um indicador significativo de saúde mental e bem-estar geral (LAHEY, 2009; NUNES et al., 2009; WATSON; CASILLAS, 2003). Por isso o presente estudo contribuiu para mostrar que as mulheres, em geral, e os homens solteiros, em particular, por vivenciarem mais neuroticismo, especialmente manifestado como sentimentos depressivos, requerem mais atenção dos profissionais da saúde, como de psicólogos que oferecem serviços de prevenção e apoio aos estudantes universitários.

Apesar desses dados comparativos entre grupos, que sugerem demandas psicológicas e alvos prioritários de intervenção em saúde na população estudada, uma análise comparativa dos escores desta amostra com a amostra original normativa da escala de neuroticismo utilizada (HUTZ; NUNES, 2001) revelou que os homens e mulheres do presente estudo apresentaram tendência geral ao neuroticismo em grau de moderado a baixo. O neuroticismo médio da amostra rondoniense se situou abaixo do ponto médio da escala normativa e acima da pontuação inferior considerada crítica. O resultado sugere que a população avaliada, talvez por um padrão cultural diferenciado da amostra normativa do instrumento, tende a enfrentar situações desafiadoras do cotidiano com menos preocupações intensas, na maioria dos casos, o que pode ser uma característica protetora da saúde.

Pode-se trabalhar com a hipótese de que os desafios enfrentados na recente ocupação regional, a construção de relações multiculturais decorrentes dos diversos fluxos migratórios, as incertezas provocadas pelos deslocamentos migratórios e a própria mobilidade social contribuíram para formar e capacitar as pessoas para o enfrentamento de desafios, o que poderia explicar o neuroticismo de moderado a baixo. Talvez numa região de formação mais antiga, com regras de comportamento social mais estabelecidas e arraigadas, as pessoas sofram mais as mudanças sociais em curso. Trata-se de uma hipótese de trabalho. No entanto, embora os escores regionais rondonienses não sejam críticos, vale lembrar que neuroticismo baixo pode significar uma tendência a não evitar riscos desnecessários (HUTZ; NUNES, 2001), de modo que seus efeitos requerem mais investigações.

No presente estudo, o neuroticismo variou em magnitude muito reduzida com a idade dos estudantes e com o nível socioeconômico dos mesmos, dispensando maior atenção para essas variáveis. De todo modo, vale apontar o estudo de Viken et al. (1994) no qual foi encontrado que o neuroticismo decresceu com a idade em mulheres. Por sua vez, Twenge (2000) observou que o neuroticismo varia mais com o ambiente na infância e adolescência, tornando-se mais estável 
na fase adulta, chamando a atenção para as experiências dos indivíduos nessas fases críticas do desenvolvimento. $O$ referido pesquisador observou, também, que o grau de neuroticismo tem aumentado de uma geração para a outra, provavelmente pela fragmentação dos vínculos sociais, sugerindo como estratégia prioritária de intervenção para a redução do neuroticismo o aumento da integração social. Estreitar laços sociais aumentaria o sentimento de segurança e reduziria o neuroticismo, destaca o autor. Futuros programas em saúde coletiva que tiverem como objetivo a redução do neuroticismo poderiam contemplar a mencionada estratégia, contribuindo para as pessoas construírem ou ampliarem suas redes de apoio social.

A Escala Fatorial de Neuroticismo - EFN (HUTZ; NUNES, 2001) tem sido a escala mais utilizada por pesquisadores no Brasil na avaliação do neuroticismo e a qualidade dos instrumentos psicológicos depende da existência e uso de normas regionalizadas para a sua adequada interpretação (SILVA; NAKANO, 2011). O presente estudo oferece os primeiros dados normativos específicos para a interpretação da EFN em adultos jovens do Estado de Rondônia.

\section{Considerações finais}

Por fim, deve ser notado que no meio universitário do Estado de Rondônia, conforme os resultados do presente estudo, as mulheres são a maioria, representando cerca de $70 \%$ dessa população. Consequentemente, em Rondônia, os postos de trabalho que exigem qualificação de nível superior serão nos próximos anos cada vez mais ocupados por mulheres, devendo chamar a atenção dos profissionais da saúde para os diferentes serviços que o público feminino irá demandar em função das exigências laborais. O dado é coerente com as estatísticas do INEP (2013) que mostram que, no ano de 2011, $60,5 \%$ dos estudantes que concluíram o ensino superior em Rondônia eram do sexo feminino. Além disso, infere-se, com os resultados do presente estudo, que a metade do público universitário rondoniense é muito jovem e que a maioria desse público é solteira e pertence às classes socioeconômicas B e C. Os dados sugerem que os estudantes universitários serão provavelmente responsáveis pela multiplicação expressiva da oferta de serviços especializados de nível superior em todo o estado, e que uma parte dele passará gradativamente por uma ascensão socioeconômica em decorrência dos investimentos atuais no ensino superior. A área das Ciências Exatas, no entanto, apareceu no estudo em menor proporção, indicando que os profissionais dessa área permanecerão escassos em Rondônia nos próximos anos, se não houver maiores investimentos e planejamento para alterar essa realidade. 
Os resultados mostraram que 0 neuroticismo variou significativamente conforme o sexo e o estado civil dos estudantes. Muito embora os resultados discutidos ofereçam alvos estratégicos para a organização e planejamento de programas de intervenção para a saúde do universitário, o presente estudo apresenta limitações. Tratou-se de uma pesquisa descritiva cujos dados não estabelecem relações causais, mas são heurísticos para 0 levantamento de hipóteses a serem investigadas em futuros estudos para a compreensão das circunstâncias psicossociais que acompanham o neuroticismo.

\section{Referências}

ALMEIDA, L. S.; GUISANDE, M. A.; SOARES, A. P.; SAAVEDRA, L. (2006). Acesso e sucesso no Ensino Superior em Portugal: Questões de gênero, origem sócio-cultural e percurso acadêmico dos alunos. Psicologia: Reflexão e Crítica, Porto Alegre, v. 19, n. 3, p. 507514, 2006.

ALMEIDA, L. S.; SOARES, A. P. C.; FERREIRA, J. A. Questionários de Vivências Acadêmicas (QVA-r): Avaliação do ajustamento dos estudantes universitários. Avaliação Psicológica, Itatiba, v. 1, n. 2, p. 81-93, 2002.

ANASTASI, A. Testes psicológicos. (Trad., Leite, D. M.). (2a a ed. revista). São Paulo: EPU, 1977. (Trabalho original publicado em 1961).

BUNCHAFT, G.; CAVAS, C. S. T. Sob medida: um guia sobre a elaboração de medidas do comportamento e suas aplicações. São Paulo: Vetor, 2002.

FIFE-SCHAW, C. Níveis de mensuração. In: Breakwell, G.; FifeSchaw, C.; Hammond, S.; Smith, J. A. (Eds.), Métodos de pesquisa em psicologia. (Trad., Elizalde, F. R.), 2010, p. 64-77. Porto Alegre: Artmed. (Trabalho original publicado em 2006).

GOTTLIEB, B. H. Selecting and planning support interventions. In: Cohen, S.; Underwood, L. G.; Gottlieb, B. H., Social support measurement and intervention. New York: Oxford University Press, 2000, p. 195-220.

GUNTHERT, K. C.; COHEN, L. H.; ARMELI, S. The role of neuroticism in daily stress and coping. Journal of Personality and Social Psychology, Washington, v. 77, n. 5, p. 1087-1100, 1999. HUTZ, C. S.; NUNES, C. H. S. S. Escala Fatorial de Ajustamento Emocional/ Neuroticismo. São Paulo: Casa do Psicólogo, 2001. IBOPE/ABEP Critério de Classificação Econômica Brasil. Disponível em: <http://www.abep.org>. Acesso em: 11 nov. 2004.

INEP Instituto Nacional de Estudos e Pesquisas Educacionais Anísio Teixeira. Disponível em: <http://portal.inep.gov.br/superior- 
censosuperior-sinopse>. Acesso em: 16 jul. 2013.

LAHEY, B. B. Public health significance of neuroticism. American Psychologist, Washington, v. 64, n. 4, p. 241-256, 2009.

LESCH, K. P. Neuroticism and serotonin: A developmental genetic perspective. In: PLOMIN, R; DEFRIES, J. C. (Eds.), Behavioural genetics in the postgenomic era. Washington: American Psychological Association, 2003, p. 389-423.

MAGNUS, K.; DIENER, E.; FUJITA, F.; PAVOT, W. Extraversion and neuroticism as predictors of objective life events: A longitudinal analysis. Journal of Personality and Social Psychology, Washington, v. 65, n. 5, p. 1046-1053, 1993.

NUNES, C. H. S.; HUTZ, C. S.; GIACOMONI, C. H. Associação entre bem-estar subjetivo e personalidade no modelo dos cinco grandes fatores. Avaliação Psicológica, Itatiba, v. 8, n. 1, p. 99-108, 2009. PASQUALI, L. Escalas psicométricas. In: Pasquali, L. (Org.), Instrumentação psicológica: fundamentos e práticas. Porto Alegre: Artmed, 2010, p. 116-135.

REIS, H. T.; COLLINS, N. Measuring relationship properties and interactions relevant to social support. In: Cohen, S.; Underwood, L. G.; Gottlieb, B. H., Social support measurement and intervention. New York: Oxford University Press, 2000, p. 136-192.

SADOCK, B. J.; SADOCK, V. A. Compêndio de psiquiatria. (Trad., Dorneles, C. O.; Monteiro, C.; Ortiz, I. S.; Cataldo, R. C.). (9a. ed.). Porto Alegre: Artmed, 2007.

SCHNEEWIND, K. A.; KUPSCH, M. Patterns of neuroticism, workfamily stress, and resources as determinants of personal distress. J ournal of I ndividual Differences, Massachusetts, v. 28, n. 3, p. 150-160, 2007.

SCOLLON, C. N.; DIENER, E. Love, work, and changes in extraversion and neuroticism over time. Journal of Personality and Social Psychology, Washington, v. 91, n. 6, p. 1152-1165, 2006.

SERPA, M. N. F.; SANTOS, A. A. A. Atuação no ensino superior: um novo campo para o psicólogo escolar. Psicologia Escolar e Educacional, Campinas, v. 5, n. 1, p. 27-36, 2001.

SILVA, I. B.; NAKANO, T. C. Modelo dos cinco grandes fatores da personalidade: análise de pesquisas. Avaliação Psicológica, Porto Alegre, v. 10, n. 1, p. 51-62, 2011.

STURGIS, P. Levantamento e amostragem. In: Breakwell, G.; FifeSchaw, C.; Hammond, S.; Smith, J. A. (Eds.), Métodos de pesquisa em psicologia. (Trad., Elizalde, F. R.), 2010, p. 115-132. Porto Alegre: Artmed. (Trabalho original publicado em 2006).

TWENGE, J. M. The age of anxiety? Birth cohort change in anxiety and neuroticism, 1952-1993. Journal of Personality and Social Psychology, v. 79, n. 6, p. 1007-1021, 2000.

VENDRAMINI, C. M. M.; SANTOS, A. A. A. DOS; POLYDORO, S. A. J.; SBARDELINI, E. T. B.; SERPA, M. N. F.; NATÁRIO, E. G. Construção e 
validação de uma escala sobre avaliação da vida acadêmica (EAVA). Estudos de Psicologia, Natal, v. 9, n. 2, p. 259-268, 2004.

VIKEN, R. J.; ROSE, R. J.; KAPRIO, J.; KOSKENVUO, M. A developmental genetic analysis of adult personality: Extraversion and neuroticism from 18 to 59 years of age. Journal of Personality and Social Psychology, Washington, v. 66, n. 4, p. 722-730, 1994. WATSON, D.; CASILLAS, A. Neuroticism: Adaptive and maladaptive features. In: CHANG, E. C.; SANNA, L. J. (Eds.), Virtue, vice, and personality: The complexity of behavior. Washington: American Psychological Association, 2003, p. 145-161.

WEINSTOCK, L. M.; WHISMAN, M. A. Neuroticism as a common feature of the depressive and anxiety disorders: A test of the revised integrative hierarchical model in a national sample. Journal of Abnormal Psychology, Washington, v. 115, n. 1, p. 68-74, 2006.

\section{Endereço para correspondência Mansueto Dal Maso}

Faculdade de Ciências Biomédicas de Cacoal - FACIMED

Av. Cuiabá, 3087 - Jd. Clodoaldo, CEP 76.963-665, Cacoal - Rondônia, Brasil

Endereço eletrônico: mansy@uol.com.br

\section{Fabio Biasotto Feitosa}

Universidade Federal de Rondônia - UNIR

Departamento de Psicologia - DEPSI, Campus - BR 364, Km 9,5, CEP 76801-059 Porto Velho - RO, Brasil

Endereço eletrônico: fabiobfeitosa@yahoo.com.br

Recebido em: 09/01/2012

Reformulado em: 16/07/2013

Aceito para publicação em: 29/09/2013

Acompanhamento do processo editorial: Deise Maria Leal Fernandes Mendes

\section{Notas}

* Graduado em Filosofia e Teologia, mestre em Ciências Sociais pela Pontifícia Universidade Católica de São Paulo - PUC e doutor em Ciências Sociais pela Universidade Estadual de Campinas - UNICAMP.

** Psicólogo graduado pela Universidade Estadual Paulista - UNESP e doutor em relações interpessoais pelo PPGEEs da Universidade Federal de São Carlos UFSCar. Fundador e coordenador do Laboratório de Relações Interpessoais e Saúde (LARIS/UNIR, <http://www.laris.unir.br>).

${ }^{1}$ Agradecimentos são endereçados a todas as pessoas e instituições envolvidas na realização do presente estudo. Apoio: CNPq.

2 De acordo com o censo do INEP <http://www.inep.gov.br/superior/censosuperior/sinopse/default.asp>, existiam no Estado de Rondônia em 2008 duas (7\%) instituições federais de ensino superior e $27(93 \%)$ instituições privadas. Considerando o número de ingressos no mesmo ano, as instituições federais representaram $16 \%(\mathrm{~N}=1.869)$ das vagas do ensino superior e as instituições privadas $84 \%(N=9.752)$. Sendo assim, a proporção da distribuição de sujeitos por tipo de instituição no presente estudo, somada ao elevado número de participantes, sugere a representatividade da amostra para a população universitária do Estado de Rondônia. 